\title{
Revising recognition judgments during noisy recognition evidence accumulation: The dynamics of losses versus gains
}

\author{
Antonio Jaeger ${ }^{1}$ - Ian G. Dobbins ${ }^{2}$
}

Published online: 22 May 2017

(C) Psychonomic Society, Inc. 2017

\begin{abstract}
Outside the laboratory, we sometimes revise our recognition judgments of others - realizing, for example, that we have accidentally failed to greet an acquaintance we just passed in the hallway. These recognition reversals have rarely been studied. Here, using a basic noisy-accumulation framework, we simulated recognition response reversals in which initial speeded recognition judgments were followed by an opportunity to revise the initial judgment. The simulation predictions were compared to empirical data from two experiments in which we gave participants the opportunity to revise each of their initial speeded recognition judgments. The speeded old-new responses were restricted to either 300 $800 \mathrm{~ms}$ (Exp. 1) or 200-600 ms (Exp. 2) after each probe's onset, and the second response was self-paced in both experiments. The noisy-accumulation framework correctly anticipated three findings. First, gain rates (incorrect followed by correct responses) always exceeded loss rates (correct followed by incorrect responses). Second, despite being corrective, the raw gain rates exhibited a modest negative correlation with overall recognition skill. Third, when gain rates were conditioned on the opportunity to correct an initial error (conditional gain rate), they were then positively correlated with recognition skill but were less diagnostic than the conditional loss rates. Thus, the mechanics of noisy accumulation naturally predict that skilled recognizers will demonstrate infrequent
\end{abstract}

Antonio Jaeger

antonio.jaeger@gmail.com

1 Department of Psychology, Federal University of Minas Gerais, Antonio Carlos Avenue, 6627, Belo Horizonte, MG 31270-901, Brazil

2 Department of Psychology, Washington University, St. Louis, MO, USA corrective behavior but a high probability of correction, should an initial error occur.

Keywords Memory $\cdot$ Recognition $\cdot$ Simulation . Accumulation

Outside the laboratory, most of us have embarrassed ourselves by accidentally failing to acknowledge an acquaintance walking by or, conversely, by warmly and informally greeting a complete stranger. Under both circumstances, an initial erroneous recognition judgment is corrected, albeit too late to avoid a social faux pas. In the present report, we focus on participant-initiated alterations of speeded recognition judgments.

These sorts of everyday memory reversals suggest that recognition evidence somehow evolves over time, with accuracy often increasing as the time given to respond also increases (Boldini, Russo, Punia, \& Avons, 2007; Gronlund \& Ratcliff, 1989; Hintzman \& Caulton, 1997; Heit, Brockdorff, \& Lamberts, 2003; Kim \& Glanzer, 1993; Reed, 1973). In general, studies investigating the putative accrual of recognition evidence use the response-signal paradigm, which provides an imperative response deadline at various intervals after recognition probes are presented. These speeding procedures are also used to investigate whether putative retrieval processes, such as contextual recollection and item familiarity, differ in their temporal onsets and/or availability (Boldini, Russo, \& Avons, 2004; Hintzman, Caulton, \& Levitin, 1998; Hintzman \& Curran, 1997; Johnson, Kounios, \& Reeder, 1994; McElree, Dolan, \& Jacoby, 1999; Yonelinas \& Jacoby, 1994).

According to dual-process models of recognition, the process of recollection typically involves the recovery of contextual information defining an episode or event (Mandler, 1980), 
whereas familiarity is thought to be a strength-like process, governed by the fluency with which stimuli themselves are identified or processed (Jacoby \& Dallas, 1981). A natural prediction from such characterizations is that recollection will be slower than familiarity to reach asymptote (Hintzman et al., 1998), and prior research involving response signal manipulations has provided support for this interpretation across different memory tasks and stimuli, including dissociation tasks (Benjamin \& Craik, 2001; Hintzman et al., 1998; Jones, 2006; Toth 1996; Yonelinas \& Jacoby, 1994, 1996), modality judgments (Hintzman \& Caulton, 1997), associative tasks (Gronlund \& Ratcliff, 1989; Rotello \& Heit, 2000), source-monitoring tasks (Gronlund, Edwards, \& Ohrt, 1997; Hintzman \& Curran, 1997; Johnson et al., 1994; McElree et al., 1999), and recognition tasks using semantically related lures (Dosher, 1984; for a review, see Yonelinas 2002).

Despite the fact that response signal paradigms suggest that the total recognition evidence increases across brief periods, they have not actually demonstrated that observers will reverse initial decisions on the basis of this accruing evidence. Thus, whereas we know that individuals given a longer response deadline will correctly identify additional studied probes as recognized, we do not know whether responders would have reached these same conclusions if they had been required to revise initial judgments that these same specific probes were new. In the language of metacognitive research, the response signal paradigm fails to illustrate whether metacognitive control (Koriat \& Goldsmith, 1996) is exerted at the level of each memory probe and is thus a poor proxy for the kinds of memory reversal experiences discussed in the opening paragraph. Moreover, whereas considerable research has suggested that increasingly speeding recognition judgments greatly limits the contributions of recollection (and, to a lesser extent, of familiarity) to recognition performance (Boldini et al., 2004; Boldini et al., 2007; Espinosa-García, Vaquero, Miliken, \& Tudela, 2017; Toth, 1996), the actual decision models often used to characterize recognition judgment are themselves "static" (e.g., Wixted \& Mickes, 2010; Yonelinas, Dobbins, Szymanski, Dhaliwal, \& King, 1996). By "static," we mean that the evidence variables assumed under these models do not evolve during the course of memory judgment; thus, these models do not address the actual dynamics of how discrimination accuracy improves over time.

In contrast to the response signal paradigms, two studies have examined the patterns of changes in judgments when observers are given two serial response opportunities for each probe (two-response paradigm). For example, Van Zandt and Maldonado-Molina (2004) allowed participants to alter their initial speeded judgments by giving them the chance to give a second recognition confidence response. In two of the four experiments reported by Van Zandt and Maldonado-Molina, the initial judgment was heavily speeded via the response-time window manipulation, and these data demonstrated that response reversals were far more likely for initially incorrect than for correct responses. For clarity, we refer to response reversals that correct initial errors as "gains" and those that mistakenly abandon initially correct reports as "losses." The tendency toward greater gains than losses did not differ as a function of the old-new status of the probes, nor was it affected by induced decision biases toward "old" or "new" initial decisions (accomplished by instruction and test list composition). Similar findings were obtained in the study reported by Curran, DeBuse, and Leynes (2007), a study in which the main departure from the study of Van Zandt and Maldonado-Molina was maintaining equal list frequencies of old and new probes, and instead manipulating response bias via the payoff matrices (see also Pleskac \& Busemeyer, 2010).

To understand the expected relationship between the four possible decision outcomes in the two-choice procedure developed by Van Zandt and Maldonado-Molina (2004), we conducted Monte Carlo simulations of a simple noisy-accumulation process. Under sequential-sampling models such as the drift-diffusion model (DDM), recognition evidence accumulates over time (Brown \& Heathcote, 2008; Reddi \& Carpenter, 2000). Indeed, the DDM was developed by Ratcliff (1978) as an attempt to account for the data provided by a variety of memory retrieval paradigms, and it has recently been used to explore differences in the recognition judgments of older versus younger adults (Ratcliff, Thapar, \& McKoon, 2004, 2010; Starns \& Ratcliff, 2010). According to this model, the evidence variable is assumed to be inherently noisy within each trial and can be conceptualized as two summed components: a linearly cumulating true signal, or "drift rate," that is contaminated by a random-walk, binomial noise process. The addition of within-trial, moment-to-moment noise is one component that generates errors within the model (cf. Brown \& Heathcote, 2008; Donkin, Averell, Brown, \& Heathcote, 2009; Reddi \& Carpenter, 2000). Ten trials of this process, using a drift rate of .15 and binomial random noise of $+3 /-3$ at each time step, are depicted in Fig. 1. On average, the slope is approximately .15 and travels in the correct direction; however, on some trials it generally falls below zero, even though the underlying drift rate is positive. In these cases, an observer would likely incorrectly conclude that the stimulus was from the negative class, and again, these errors are triggered by the cumulative effects of random noise within the trial.

Next we expanded the simulation to 75 trials and isolated four different possible two-response outcomes, by contrasting the mean evidence value over the first 250 time points with the mean evidence value over the last 250 time points. These two intervals were used to represent two serial epochs that an observer might use during a two-choice recognition paradigm. They do not represent literal time, but form a starting point for understanding the dynamics of the average of the noisy evidence variable in two serial epochs of equal duration. If the 


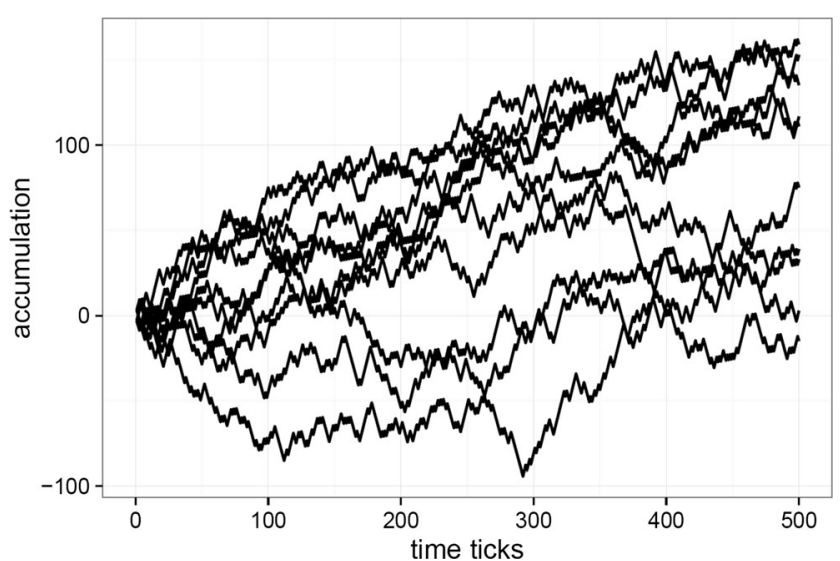

Fig. 1 Evidence accumulation for ten trials and a positive drift rate of .15 (binomial noise +3 or -3 over 500 time ticks)

mean in the first epoch is positive, we assume that the observer would likely conclude that the item was from the positive class, which is a "hit." Conversely, if the mean evidence is negative, we assume the observer would conclude that the item was from the negative class, which is a "miss," because the underlying drift rate is positive. Thus, in the following illustrations, positive mean evidence yields hits, and negative mean evidence yields misses. If the mean values in the two epochs are both positive or both negative, the fictive participant responds consistently (hit-hit or miss-miss). In contrast, if the initial mean is negative and the second positive, the observer responds "new" on the initial judgment and "old" on the second judgment (miss-hit). This is a gain, since an initial error is corrected. The opposite, a hit-miss, is a loss, because an initially correct report is abandoned. The time courses of the subsets of gains and losses from the 75 trials are shown in Fig. 2a, which clearly shows that gains outnumber losses in this instance.

The tendency for gains to considerably outnumber losses is an inherent property of this type of noisy evidence accumulation, because it becomes increasingly hard to trigger errors across time, since the true underlying signal is always increasing. Figure $2 \mathrm{~b}$ more concretely illustrates this by removing the signal from the gains and losses across 2,000 trials and plotting the average cumulative noise for these reversals. The color line near 0 confirms that across all trials, cumulative noise is neutral, as expected. In contrast, gains result from uncharacteristically negative noise in the first epoch (yielding a miss), followed by more neutral noise in the second epoch (enabling a hit). Losses result from neutral and then modestly negative noise in the first epoch (yielding a hit), followed by extremely negative noise in the second epoch (yielding a miss). The comparison of the gain versus the loss noise profiles demonstrates why losses are rarer than gains: They require more improbable noise behavior. On very few trials will random noise continue to systematically negatively accumulate as is required to induce a loss.
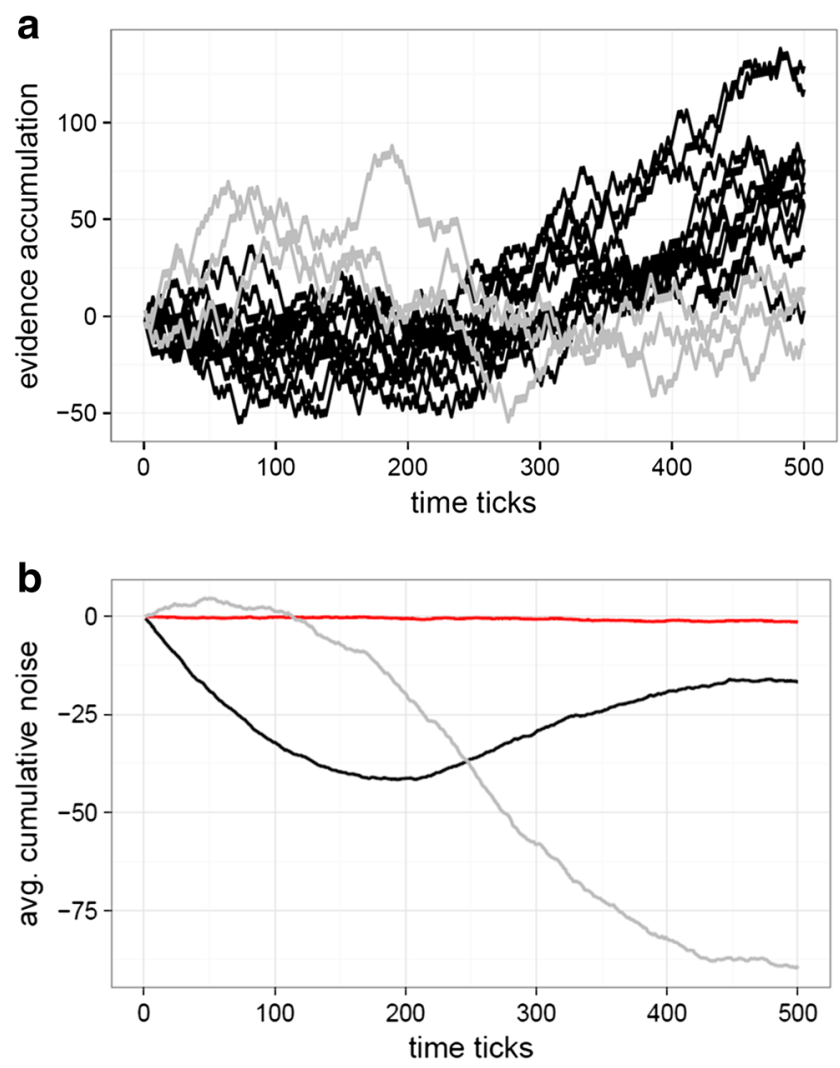

Fig. 2 (a) Gains (black) versus losses (gray) in an evidence accumulation process using equal epochs. (b) Average profiles of cumulative noise for gains (black) versus losses (gray).

\section{Raw outcome proportions}

To calculate reversal proportions, we next examined 2,000 trials, now using drift rates that would generate from chance to perfect overall performance with respect to the proportion of correct final responses (slopes/rates of .01 to .4). For each drift rate, the four possible serial outcomes [i.e., hit-hit, missmiss, miss-hit (gain), and hit-miss (loss)] were tallied. We focus on gains and losses, and these rates are plotted against the final overall proportion correct of each fictive observer with a different underlying drift rate. Critically, under this simplified noisy-accumulation model, the final proportion correct is a near linear indicator of the underlying drift rate of the observer. Thus, Fig. 3 captures the relationships between reversal behavior rates and the underlying drift rates generating the data, but it does so using an observable value - namely, the overall proportion correct as a proxy for the actual underlying drift rate. For generality, the simulations considered three levels of Response 1 relative to Response 2 speeding, with the top panel replicating the equal-interval scenario examined earlier (both intervals of 250 time ticks). The second panel speeds the initial judgment somewhat, by reducing the first epoch to 150 time ticks and increasing the second to the remaining 350 time ticks. Finally, the last panel shows the initial performance speeded even further, such that the first 

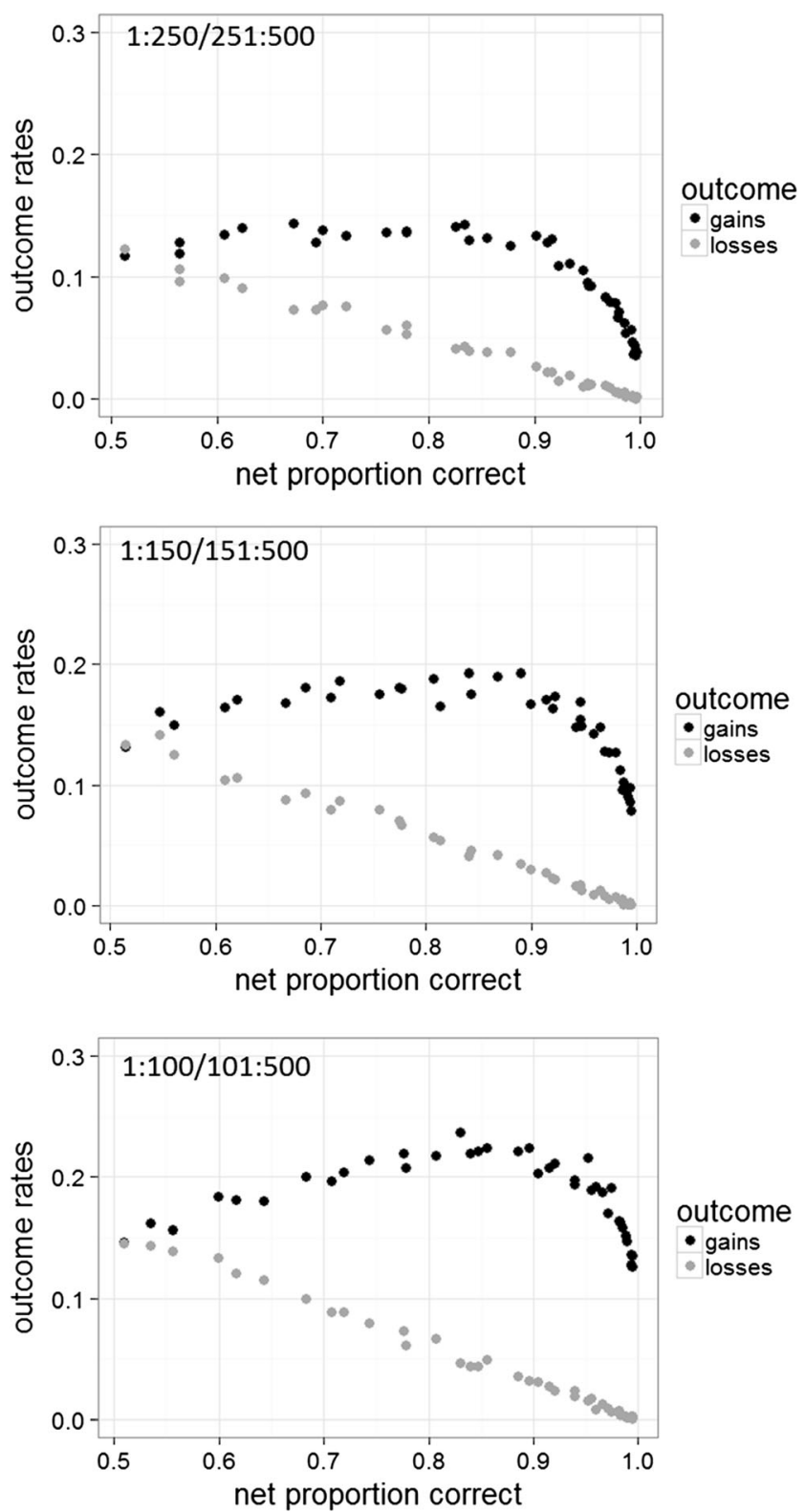

Fig. 3 Monte Carlo simulations of the relationship between gains, losses, and the final or net proportion of correct responses in the two-response paradigm. Losses indicate a correct followed by an incorrect response, whereas gains indicate an incorrect followed by a correct response. The top panel shows outcomes when the first and second means are based on equal epochs. The middle panel uses the first 150 observations for the first mean and the remaining 350 observations for the second mean. The bottom panel uses the first 100 observations for the first mean and the final 400 observations for the second. The relative spans of the first and second responses are shown in the inset within each panel. Thus, the topto-bottom differences capture an observer whose first response is increasingly sped.

response is based in the initial 100 time ticks, and the second response, the remaining 400 time ticks.

In all three simulations, unsurprisingly, losses are negatively associated with the overall proportion correct (and hence, the underlying drift rate); as overall skill increases, the raw loss rate declines (gray). Thus, as the drift rate increases, it becomes highly unlikely to observe a positive followed by a negative mean signal across the epochs. However, the relationship between the raw gain rates and overall success is more surprising: It begins fairly flat and then displays a prominent downward trend as overall performance climbs past somewhere in the range of .8 to .9 . This relationship is somewhat surprising because one might expect that observers who are good at correcting initial errors would have high overall performance rates; that is, one might expect there to be a positive relationship between gain rates and overall success rates, at least throughout most of the range of overall ability. However, two opposing forces influence the observed raw gain rate. As the drift rate increases, it becomes increasingly unlikely that the observers will miss the first judgment, and hence the opportunities to observe gain trials decrease, driving down the raw gain rates. However, as the drift rate increases, it also becomes increasingly likely that, should a miss occur on the first judgment, it will be corrected on the second, and this tends to drive up the raw gain rates. These oppositional tendencies balance throughout most of the range of performance, causing a flat to slight positive relationship, until the overall performance exceeds approximately .8 to .9 , at which point the drift rates become so large, relative to the inherent noise, that erring on the first response begins to drop dramatically. Thus, the raw rate of correcting initial errors under this model is not a marker of skilled recognition. In fact, as we show below using small- $N$ simulations, the raw gain rates are anticipated to be negatively correlated with success rates across individuals, consistent with the negative downward terminal bend in all three gain rate curves in Fig. 3. To our knowledge, this is the first time the noisy-accumulation framework has been used to predict relationships in a two-response recognition procedure at the level of individual differences.

\section{Conditional gains and losses}

The relatively flat, then downward, relationship between raw gain rates and proportions correct in Fig. 3 results from the geometry of the accumulation model. However, it would be incorrect to assume that the flat-to-negative relationship in raw outcome proportions means that gains are not somehow positively linked to the underlying drift rates. As we noted above, whereas an increasing drift rate will reduce initial errors (lowering the possibility for raw gains), it will necessarily also increase the probability that an initial error will be corrected, should it occur. This is the conditional gain rate (Fig. 4). Here the raw gains and losses are conditioned upon the first response outcome. Thus, the conditional gain rate is the raw gain rate divided by this rate plus the raw miss-miss rate. This normalizes the raw gain rate by the total opportunity to observe a gain - that is, by the total proportion of misses on 

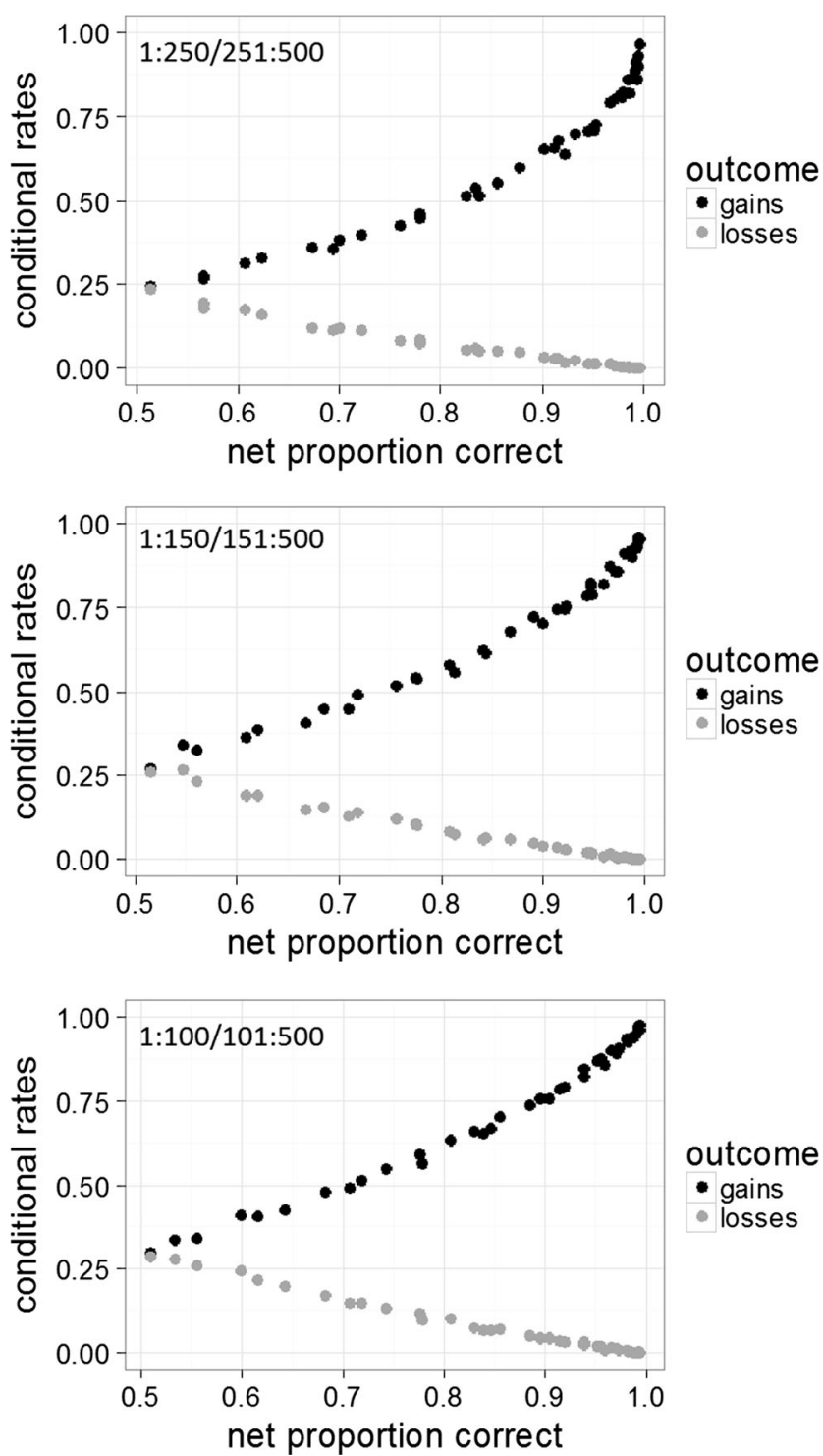

Fig. 4 Monte Carlo simulations of the relationship between both conditional gains and losses and the final proportion of correct responses in the two-response paradigm. As in Fig. 3, the three panels reflect increasing speeding of the first response from top to bottom, with the relative durations of the first and second response periods illustrated in each panel using text insets.

Response 1. Analogously, the conditional loss rate normalizes the raw loss rate by the total proportion of hits in the first response (loss rate plus hit-hit rate), and thus the conditional loss rate is the proportion of initial hits that yield errors on the second response. Plotted in this manner, it becomes clear that conditional gain rates positively reflect the underlying drift rate, and hence the final overall performance. Unsurprisingly, conditional losses remain highly negatively predictive of overall performance. The fact that conditional gains are positively linked to performance is not surprising in and of itself. Instead, the fundamentally different nature of this relationship in comparison to the raw gain rates is what is important. Raw gain rates are negatively associated with skill
(Fig. 3), whereas conditionalized gain rates are positively associated (Fig. 4). Moreover, Fig. 4 also shows that the relationship between conditional gains and performance is not as tight as that between conditional losses and performance. This can be appreciated by considering the ranges of the two conditional scores relative to the proportion correct. Whereas conditional losses range from .25 to 0 as the proportion correct ranges from chance to perfect, conditional gains range from about .25 to 1 as the proportion correct ranges from chance to perfect. In other words, a small change in conditional losses predicts a greater change in overall performance across subjects than does the same small change in conditional gains. Thus, even when both scores are conditionalized, losses remain more sensitive than gains to individual differences in skill.

\section{Small-sample predictions}

The simulations in Figs. 3 and 4 are essentially noise-free. That is, every sampled drift rate spanning chance to perfect overall performance (40 total) was applied in 2,000 trials to estimate the response rates. In contrast, the observers in the empirical data reported below received trial counts ranging from 60 to 160, depending upon the item class, a range consistent with typical recognition memory studies. Moreover, a high density of simulation observations are above the overall proportion correct of $\sim .9$, which results from the facts that drift rates were evenly sampled but the relationship between drift rate and proportion correct becomes nonlinear above $\sim .9 .{ }^{1}$

Thus, to get a firmer prediction of the anticipated empirical relationships, we reran the simulations with only 75 trials per sampled drift rate for 300 replications. Additionally, on each replication we randomly selected 25 of the 40 simulated drift rates with replacement. This is analogous to randomly sampling 25 participants with potentially widely varying abilities without constraining how their overall ability is distributed within the sample. In other words, this assumes that the distribution of performance in each of the 300 small samples of 25 may be quite "lumpy." Within each of the 300 replications of the 25 fictive participants, we recorded correlations between both the gain and loss rates (raw and conditional) and

\footnotetext{
${ }^{1}$ The actual correlation between the simulation drift rates and proportions correct (hit-hit + gain rates) was .93. As the figure in the Appendix shows, the relationship between the underlying drift rate and the observed proportion correct is roughly linear until the latter reaches about .95 , at which point increases in the drift rate yield diminishing returns in terms of proportions correct. Using the $z$ score of the proportion correct instead of the raw value increased the relationships to .99 , indicating that the underlying drift rate is effectively the $z$ score of the observed proportion correct under this simple noisy-accumulation model (see also Ratcliff \& McKoon, 2008). Nonetheless, we focus on the untransformed proportions correct (a) for ease of exposition, (b) because the relationship is quite near linear below .95, and (c) to avoid difficulties with perfect performance, since the $z$ transform is undefined for proportions of 1 , which occurred regularly with deep encoding.
} 
the overall proportion correct. Thus, the spread and locations of the correlations across the 300 replications constitute the empirical data predictions under the model for small-sample designs. It is important to note that the use of correlation coefficients does not assume that the underlying relationship between the rates and overall performance is linear, which is clearly not the case for raw gain rates in Fig. 3. Instead, the simulation anticipates how correlation coefficients should behave given the model dynamics in Fig. 3. Therefore, although it is improper to assert that an underlying relationship must be linear given an observed correlation coefficient, it is valid to predict the behavior of correlation coefficients, given a nonlinear underlying model generating the data.

Figure 5 illustrates the anticipated behavior of correlations of both gains and losses with overall performance, when gains and losses are either raw or conditioned. Beginning with raw and conditional loss rates, Fig. 5 demonstrates that these are both highly negatively correlated with overall performance, regardless of the degree of initial response speeding. Although it is difficult to see because of the overlap of the histograms, the conditional losses tended to be slightly more strongly negatively correlated with overall performance than the raw losses. Nonetheless, the key point is that both are strongly negatively correlated in this small $-N$ scenario. In contrast, a fundamentally different pattern emerges for raw and conditional gains. The former are generally negatively correlated with performance across the fictive participants. Thus, despite the increased variability resulting from the small trial count (75) and the small number of sampled participants (25), the expectation is that raw gains will be modestly negatively linked to overall performance. That is, participants who correct more errors are expected to be less accurate overall. Turning to the conditional gains, however, the simulation reveals that these are predicted to strongly positively correlate with overall performance, without exception. Moreover, the simulation shows that the sampling distributions of gain correlations and those of conditional gain correlations are wholly nonoverlapping. ${ }^{2}$ Thus, they should be significantly different in empirical data in the anticipated directions.

The histograms also demonstrate that raw and conditional gains shift rightward as the first response is increasingly sped, a pattern that is most noticeable for the raw gains. This occurs because as the first response is increasingly sped, the contribution of the drift rate increasingly shifts from the first to the second epoch. In the extremes, first responses are driven almost entirely by noise, and thus responding above chance overall would depend solely on the expression of the drift rate during the second response period. Thus, since speeding renders the second response increasingly linked to the drift rate, and since the overall proportion correct is determined by the

\footnotetext{
$\overline{2}$ These predictions for gains, losses, and overall performance remain the same, regardless of whether Pearson's $r$ or Spearman's rho is used.
}

drift rate, the correlation between gain behavior and proportion correct increases.

\section{Summary}

The basic noisy-accumulation model makes the following new predictions about the relationship between gains, losses, and overall performance within and across individuals:

1. Raw gain rates will exceed raw loss rates. Under the mod$\mathrm{el}$, this is due to the behavior of cumulative noise within the trial. Losses require noise that is much more biased and unusual than gains, and so they are rarer.

2. Raw gain rates will be negatively associated with individual differences in overall skill. Although raw gains constitute corrections, the fact that these corrections are necessary suggest low signal to noise across the trials of the observer. This in turns means that one should observe a divergence such that raw gains are negatively associated with individual differences in skill, whereas conditional gains are positively associated with individual differences in skill.

3. Although conditional gains are positively linked to individual skill, they should be less reliably so, in an absolute sense, than conditional losses, which are negatively associated with skill (Fig. 4). Thus, the correlations between conditional loss rates and overall performance, although negative, should be larger in absolute value than the correlations between conditional gain rates and overall performance.

\section{Experiment 1}

In the present study, we implemented the two-response recognition paradigm. Additionally, to broaden the range of performance outcomes, the studied materials were encoded with either a pleasantness judgment or a syllable-counting task (deep or shallow encoding, respectively). During testing, participants gave two serial classifications of each item. The first response consisted of a simple old-new decision, and the time allowed for its execution was restricted to within $300-800 \mathrm{~ms}$ from the presentation of each item. Following this, participants performed a second, self-paced old-new recognition judgment on the same words. They were encouraged to correct mistaken first responses by giving different responses on the second opportunity.

\section{Method}

Participants Experiment 1 included 26 participants (18-22 years old; 14 females, 12 males). Informed consent was approved by the Institutional Review Board of Washington 
loss/pc correlations
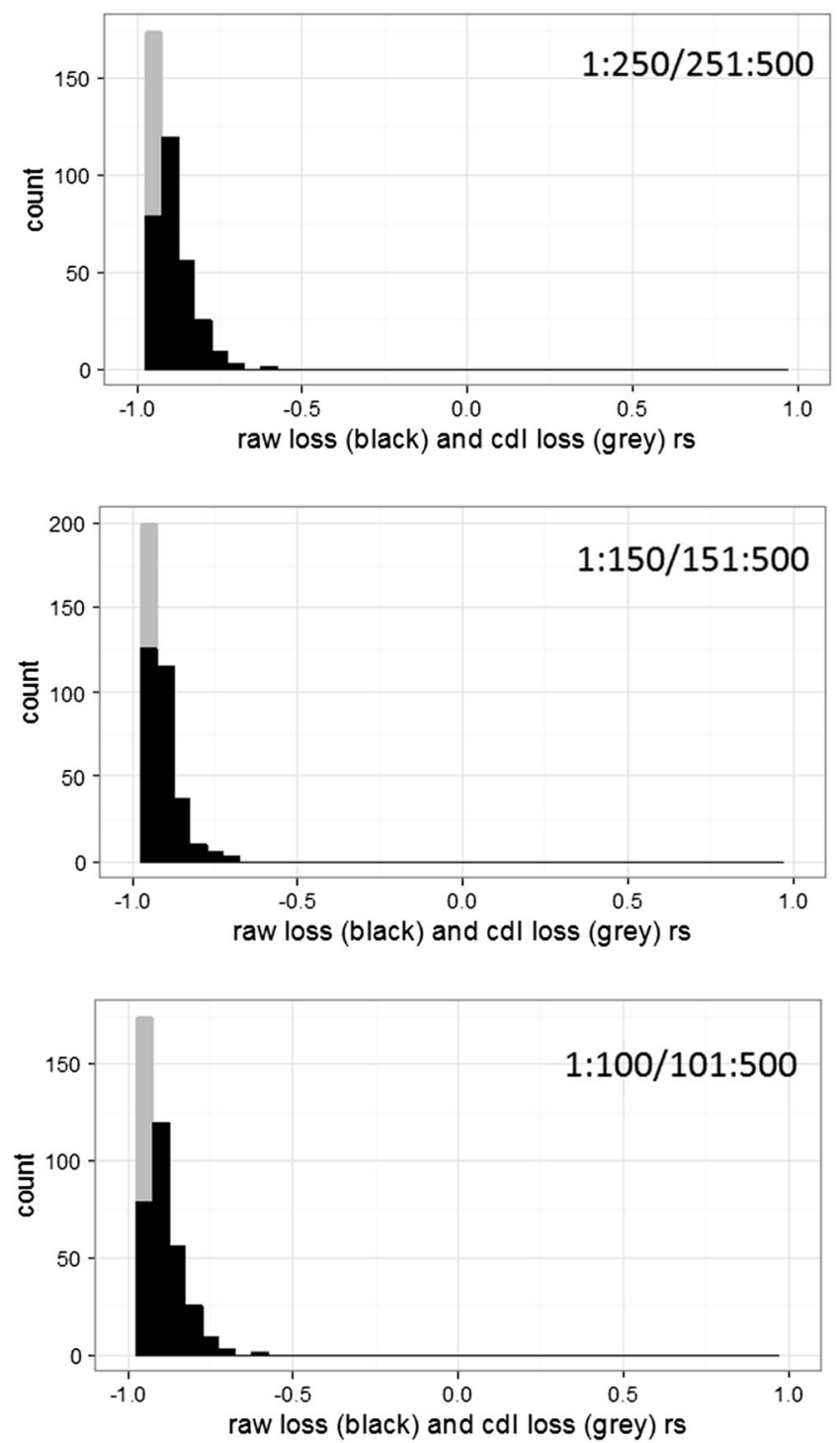

Fig. 5 Histograms showing the distribution of correlations observed in small- $N$ simulations of the noisy-accumulation model using 75 trials/per drift rate across 25 randomly sampled fictive participants per replication (300 replications). Each histogram captures the expected location and spread of correlation coefficients between loss and gain rates (either raw

University in St. Louis. All participants were able to keep over $50 \%$ of the speeded responses within the 300 - to 800 -ms interval, so no participant was removed from the analysis due to excessively early or late responding. Two participants were excluded due to performance at chance on both the first and second responses. One participant was excluded due to an absolute absence of reversed responses, and one due to computer failure during task execution, leaving 22 participants for the statistical analysis.

Materials A total of 320 words were drawn randomly for each participant from a pool of 1,216 words. From the list, four lists

\section{gain/pc correlations}
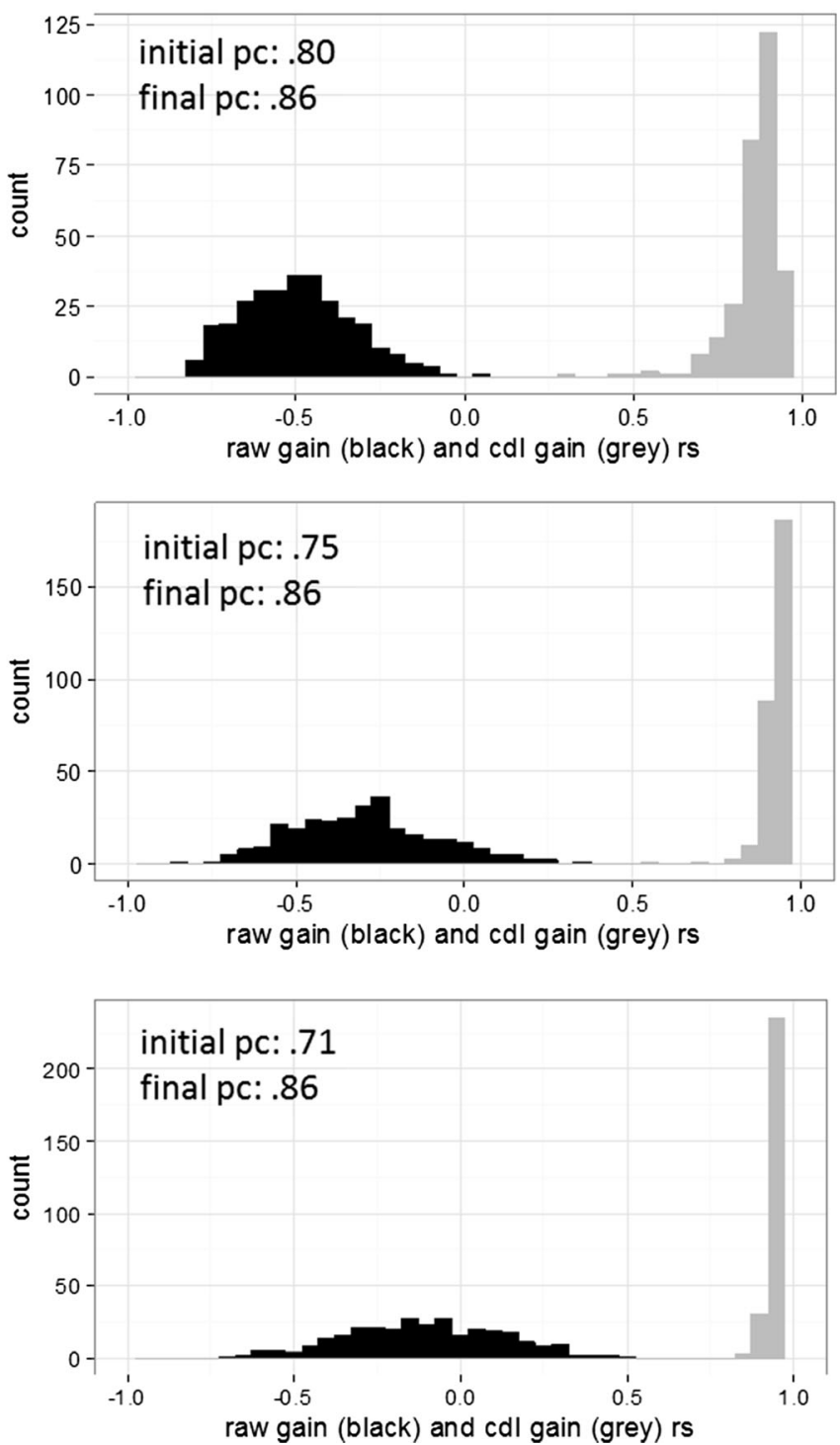

or conditioned) and observer overall performance across 300 replications. Of the 40 drift rates simulated in each replication, 25 were randomly sampled with replacement in order to estimate each correlation. This simulates random sampling of fictive participants with differing overall abilities.

of 80 items ( 40 old and 40 new for each cycle) were used in four study-test cycles. The items in the pool had, on average, 7.09 letters and 2.34 syllables, with a Kučera-Francis corpus frequency of 8.85 (Kučera \& Francis, 1967).

Procedures A maximum of four participants were tested in a room together per session. Computers were used for the presentation of stimuli and the collection of responses. The practice phase was identical to the actual experiment, except that it included only two study-test cycles of five study and ten test trials each. 
To manipulate levels of processing at study, participants were instructed that in some parts of the experiment they would be required to perform a syllable-counting task (shallow) for each word, whereas in other parts they would be required to perform a pleasantness rating task (deep) for the words. The syllable-counting task consisted of indicating whether or not each word had fewer than three syllables ("2 or fewer?"), whereas the pleasantness rating task required indicating whether each word was pleasant or unpleasant. For both encoding tasks, participants were instructed to use the "1" key to indicate the affirmative and the " 2 " key to indicate the negative. Responses were self-paced, and a 1-s fixation cross warned of the onset of the next item. Deep and shallow study-test cycles alternated across the session, with the level of processing of the first cycle counterbalanced across participants.

In each recognition memory test, studied items were intermixed with new items. Each test item was followed by a response signal $300 \mathrm{~ms}$ after its presentation. The words were first presented in white font, and the response signal consisted of the words turning red. Participants were instructed to perform an old/new judgment within $500 \mathrm{~ms}$ after the response signal onset, and they were informed that the time to respond expired when the red-colored word disappeared. To increase the number of responses within such a constrained period, participants were presented with two sequences of fixation crosses before each trial to establish the response timing. The presentation timing of the fixation mirrored that of the upcoming word presentation (a white-colored cross turning red after $300 \mathrm{~ms}$ and offsetting $500 \mathrm{~ms}$ later). This gave participants a temporal cue for the timing constraint of the upcoming memory judgment. After the initial speeded response, the memory probe was removed and followed by a blank screen (1,500 ms), and then the probe reappeared. This was the participant's cue to render the 2nd self-paced recognition judgment, and again we encouraged participants to reverse their responses if they believed that their first response was incorrect. Both responses were made by pressing the "c" or the " $\mathrm{m}$ " key (with the left or the right index finger, respectively) to indicate "old" or "new." The assignments of keys were counterbalanced across participants.

\section{Results and discussion}

Nonresponses Responses outside the 300- to 800-ms window were considered "nonresponses" and discarded from the analysis. Because participants were heavily speeded during Response 1, we first considered whether the nonresponse rates varied according to the item classes. On average, participants failed to respond within the time limit for $13 \%$ of the test probes; however, the rates did not differ across materials [deep vs. shallow probes, $t(21)=1.48, p>.15$; new items in the deep vs. shallow test lists, $t(21)=1.28, p>.20$ ]. Thus, the differences below are not a function of differential inclusion of the different classes of items across the deep and shallow tests. Nonresponse data were excluded from the remaining analyses.

Accuracy improvements As a manipulation check, we verified whether accuracy reliably improved across the first and second responses when these were completed. Although levels of processing obviously cannot be manipulated for new items, the present blocked design meant that there were two classes of new materials - namely, those tested in the context of deeply processed targets, and those tested in the context of shallowly processed targets.

Initially we sought to verify whether accuracy improved with a 2nd response opportunity and to see whether this improvement was more robust for deep than for shallow test lists. Thus, the dependent variable was the signal detection accuracy estimate, $d^{\prime}$. Because five participants exhibited perfect performance in at least one condition during the 2 nd response, to calculate $d^{\prime}$ we added .5 to each hit and false alarm frequency cell and divided it by the total number of either old or new responses plus 1, as recommended by Snodgrass and Corwin (1988) and Hautus (1995). A two-way repeated measures analysis of variance (ANOVA) with the factors response (1st vs. 2nd) and depth (deep vs. shallow) supported the idea of greater evidence for deep materials when using the $d$ 'score of each participant (Table 1). Although secondresponse (mean $d^{\prime}=2.58, S D=1.00$ ) was generally higher than first-response (mean $\left.d^{\prime}=1.72, S D=0.72\right)$ accuracy, $F(1$, $21)=79.74$, MSE $=.185, p<.001$, and deep $\left(\right.$ mean $d^{\prime}=2.54$, $S D=1.02$ ) was generally greater than shallow (mean $d^{\prime}=$ $1.77, S D=0.76)$ test accuracy, $F(1,21)=59.32, M S E=$ $.217, p<.001$, these factors also interacted, $F(1,21)=$ $69.44, M S E=.020, p<.001$. The interaction resulted because the gains in $d^{\prime}$ from the first to the second response were more dramatic for the deep than for the shallow tests. Proportions of correct responses, along with measures of accuracy and response bias, are shown in Table 1. The raw frequencies of response reversals are shown in Table 2.

Individual differences model predictions The correlations between both the raw and conditional gain rates and overall performance are listed in Table 3. The first three rows consider each item type separately, whereas the last row collapses across all three. Because the noisy-accumulation model predictions are necessarily contingent upon participants performing above chance, participants with overall performance rates below .5 were removed from each relevant analysis, as is reflected in the $N$ column of the table. This occurred once apiece for new and deep items, and twice for shallow items. It never occurred when the data were collapsed across the three item types. 
Table 1 Mean proportions of "old" responses for old and new items, accuracy $(d$ '), and response bias $(C)$ for the first and second response opportunities in Experiments 1 and 2

\begin{tabular}{|c|c|c|c|c|}
\hline & \multicolumn{2}{|l|}{ Experiment 1} & \multicolumn{2}{|l|}{ Experiment 2} \\
\hline & 1st Response & 2nd Response & 1st Response & 2nd Response \\
\hline Hits deep & $.79(.17)$ & $.89(.12)$ & $.78(.15)$ & $.94(.08)$ \\
\hline Hits shallow & $.67(.21)$ & $.74(.17)$ & $.76(.15)$ & $.81(.14)$ \\
\hline FAs "deep" & $.20(.18)$ & $.11(.13)$ & - & - \\
\hline FAs "shallow" & $.23(.17)$ & $.17(.16)$ & - & - \\
\hline FAs "intermixed" & - & - & $.42(.19)$ & $.07(.08)$ \\
\hline$d^{\prime}$ deep & $1.86(.81)$ & $2.93(1.05)$ & $1.13(0.92)$ & $3.29(0.90)$ \\
\hline$d$ shallow & $1.34(.72)$ & $1.91(.90)$ & $1.05(0.79)$ & $2.56(0.76)$ \\
\hline$C$ deep & $.02(.45)$ & $.02(.40)$ & $-.30(.36)$ & $-.03(.32)$ \\
\hline$C$ shallow & $.17(.48)$ & $.22(.42)$ & $-.26(.38)$ & $.34(.35)$ \\
\hline
\end{tabular}

Standard deviation are in parentheses. deep = deep-encoding condition; shallow = shallow-encoding condition; "deep," "shallow," and "intermixed" = new items tested in deep, shallow, and intermixed test blocks, respectively; FAs = false alarms; $d$ " = signal detection index of accuracy; $C=$ signal detection index of response bias.

Beginning with the raw and conditional gains, Table 3 supports the model. In every case, the raw-gain correlations are negative, yet the conditional-gain correlations are positive. The $95 \%$ confidence interval following the conditional-gain correlation coefficients is for the difference between the conditional-gain and the raw-gain correlation coefficients. This is a percentile-bootstrapped confidence interval based on 1,000 replications. In every case, the difference between the conditional correlation coefficients and the raw correlation coefficients is highly reliable, as is indicated by the lower bound of the confidence interval and consistent with the predictions of Fig. 5. Turning to losses, Table 3 illustrates that both the raw and conditional correlations with performance are negative. The columns after the conditional-loss data show the $95 \%$ confidence intervals for the differences between the conditional-loss and raw-loss correlation coefficients. These indicate reliable differences for new items, for shallow items, and when the three item classes are collapsed (total); however, the difference is not reliable for deep items. This less robust difference in correlation coefficients (as compared to gains) is also consistent with the predictions of Fig. 5, which shows the raw- and conditional-loss correlations bunched up against the lower bound of -1 . Finally, in three of the four comparisons, the conditional loss rate is more tightly associated with individual differences in performance (although negative in sign) than the conditional gain rate. The only comparison in which this was not true was for deep materials, but this did not recur in Experiment 2, so we do not interpret it further.

Overall, the empirical data support the model predictions. Observers with higher raw gain rates are less accurate overall, whereas those with higher conditional gain rates are more accurate overall. The differences between these negative and positive correlations are quite reliable. In contrast, both the raw- and conditional-loss correlations with performance are negative and similar in their overall magnitudes, although the data suggest that conditional loss rates are modestly more negatively correlated with performance than are raw loss rates. Finally, in three of the four comparisons, the conditional loss rate was more negatively associated with performance than the conditional gain rate was positively associated with performance. Despite the strong correspondence with the model predictions, Table 3 is not perfectly consistent with the model, in that the raw- and conditional-loss correlations are perhaps not as extreme as expected. In particular, the negative correlation between raw losses and performance for shallow materials was -.37 , whereas the simulations in Fig. 5 never produced correlations below - .5. However, this was the condition in which we removed two participants from the data for

Table 2 Raw frequencies for each reversal type

\begin{tabular}{|c|c|c|c|c|c|c|c|c|}
\hline & \multicolumn{4}{|l|}{ Experiment 1} & \multicolumn{4}{|c|}{ Experiment 2} \\
\hline & Hit-Miss & Miss-Hit & CR-FA & $\mathrm{FA}-\mathrm{CR}$ & Hit-Miss & Miss-Hit & CR-FA & $\mathrm{FA}-\mathrm{CR}$ \\
\hline Deep & $1.23(1.72)$ & $8.91(5.12)$ & $1.18(1.53)$ & $6.50(3.69)$ & $1.10(1.35)$ & $9.29(7.67)$ & - & - \\
\hline Shallow & $3.00(2.53)$ & $8.23(5.09)$ & $2.00(2.89)$ & $6.41(4.41)$ & $4.52(4.07)$ & $6.68(5.51)$ & - & - \\
\hline Intermixed & - & - & - & - & - & - & $0.87(2.70)$ & $15.87(9.42)$ \\
\hline
\end{tabular}

Standard deviation are in parentheses. Hit-Miss = reversal from hit to miss, Miss-Hit = reversal from miss to hit, CR-FA = reversal from correct rejection to false alarm, FA-CR = reversal from false alarm to correct rejection, Deep $=$ old items deeply encoded or new items within deep test blocks, Shallow $=$ old items shallowly encoded or new items within shallow test blocks, Intermixed = new items intermixed with deep and shallow studied items at test. 
Table 3 Correlations of both gains and losses with overall performance in Experiment 1

\begin{tabular}{|c|c|c|c|c|c|c|c|c|c|c|c|}
\hline \multirow{2}{*}{$\frac{\text { Items }}{\text { New }}$} & \multirow{2}{*}{$\frac{\text { Gain_r }}{-.52}$} & \multirow{2}{*}{$\frac{\text { Cdl_gain_r }}{.56}$} & \multicolumn{2}{|c|}{$95 \% \mathrm{CI}$} & \multirow{2}{*}{$\frac{\text { Loss } \_r}{-.83}$} & \multirow{2}{*}{$\frac{\text { Cdl_loss_r } r}{-.85}$} & \multicolumn{2}{|c|}{$95 \% \mathrm{CI}$} & \multirow{2}{*}{$\begin{array}{l}\text { Initialpc } \\
.81\end{array}$} & \multirow{2}{*}{$\begin{array}{l}\text { Finalpc } \\
.88\end{array}$} & \multirow{2}{*}{$\frac{N}{21}$} \\
\hline & & & .66 & 1.44 & & & -.13 & .03 & & & \\
\hline Shal & -.48 & .46 & .59 & 1.21 & -.37 & -.61 & -.43 & -.08 & .73 & .79 & 20 \\
\hline Deep & -.38 & .88 & .91 & 1.59 & -.53 & -.57 & -.08 & 0 & .82 & .92 & 21 \\
\hline All & -.37 & .67 & .68 & 1.37 & -.64 & -.71 & -.19 & -.01 & .76 & .84 & 22 \\
\hline
\end{tabular}

95\% confidence intervals are for the differences between the conditional and raw correlation coefficients. Gain $r=$ correlation between raw gain rate and proportion correct, Cdl_gain_r $r=$ correlation between conditional gain rate and proportion correct, Loss_ $r=$ correlation between raw loss rate and

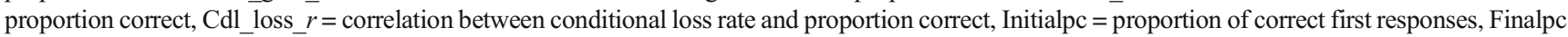
$=$ overall proportion correct.

below-chance performance, and hence it had the smallest $N$ (i.e., $N=20$ ).

\section{Experiment 2}

In Experiment 2 we attempted to replicate the findings of Experiment 1 in a design in which deep, shallow, and new items were fully intermixed at test, precluding the potential development of different decision biases for the materials. Additionally, we increased the time pressure of the first response, which should elevate the raw gain rates under the noisy-accumulation model, which were a key focus of this study. Except where noted, the methods were identical to those of Experiment 1.

\section{Method}

Participant Experiment 2 included 34 participants (18-22 years old; 17 females, 17 males). Two participants failed to keep at least one third of their speeded responses within the 200- to 600-ms interval after probe onset and were excluded from the analysis. One participant was excluded due to performance at chance on both the first and second responses. This left 31 participants for the statistical analysis.

Materials A total of 180 words were drawn randomly for each participant, to form three lists of 60 items ( 20 old deep, 20 old shallow, and 20 new items for each cycle) used in three studytest cycles.

Procedures The practice phase consisted of one study-test cycle of 20 study and 30 test trials. In contrast to Experiment 1, the level of processing at encoding was manipulated on a trial-by-trial basis via a prompt positioned on the top of the screen indicating the nature of the task to be performed ("pleasant word?" for deep encoding and "two or fewer syllables?" for shallow encoding) for each item. At test, each memory probe was followed by the response signal $200 \mathrm{~ms}$ after its onset. Participants were instructed to perform the first old/new judgment within $400 \mathrm{~ms}$ after the response signal onset. Once the time window for the first response had expired, the word stayed on the screen for $800 \mathrm{~ms}$, and the participants were not required to perform any task. After this interval, a second signal ("respond again") was shown on the screen, and a second judgment about the status of the word was made in a self-paced manner.

\section{Results and discussion}

Nonresponses Responses outside the 200- to 600-ms window were considered "nonresponses" and discarded from the analysis. Again, we initially considered the nonresponse rates for the different item classes to ensure that the key comparisons below were not biased by different numbers of items entering into analyses of the actual responses. Unlike in Experiment 1, a one-way ANOVA contrasting deep, shallow, and new materials did yield significance, $F(2,60)=14.48, p<.001$, partial $\eta^{2}=.33$. However, post-hoc comparisons demonstrated that this occurred because nonresponse rates to new materials (.31) were higher than nonresponse rates to deep (.23) and shallow (.24) materials $(p s<.001)$, which were statistically indistinguishable, $t(30)<1$. The cause for the selective elevation of nonresponse rates to new materials here as compared to Experiment 1 is unclear, since the rise could have reflected the full intermixing of the three item classes and/or the quicker response window used here. Although it does suggest an interesting difference between the studied and unstudied materials, it did not confound the analyses below, which restricted direct comparisons to the deep and shallow materials, which were well-matched in this respect.

Accuracy improvements As in Experiment 1, we compared the accuracies for deep versus shallow targets across the initial versus the subsequent response (see Tables 1 and 2). Although we used the $d^{\prime}$ accuracy measure for consistency, it should be noted that the false alarm rate was the same for each participant's deep and shallow conditions, since the deep and shallow targets were intermixed within the same test list. Thus, any accuracy difference obtained below must necessarily be a consequence of differences in hit rates. 
The adjustment for extreme performance proposed by Snodgrass and Corwin (1988) and Hautus (1995) was used in the data of all participants, since 11 participants exhibited perfect performance in at least one condition during the 2 nd response, and two during the 1st response. A two-way repeated measures ANOVA with the factors response (1st vs. 2nd) and depth (deep vs. shallow) converged with that in Experiment 1 when applied to the equal-variance $d$ ' score of each participant. Although second-response accuracy (mean $d^{\prime}=2.83, S D=0.85$ ) was generally higher than first-response accuracy (mean $d^{\prime}=1.08, S D=0.83$ ), $F(1,30)=168.77$, MSE $=.561, p<.001$, and deep (mean $d^{\prime}=2.16, S D=1.36$ ) was generally greater than shallow (mean $d^{\prime}=1.75, S D=1.02$ ) test accuracy, $F(1,30)=47.66, M S E=.111, p<.001$, these factors also interacted, $F(1,30)=23.51, M S E=.143, p<.001$. The interaction resulted because the gains in $d^{\prime}$ from first to second responses were more dramatic for the deep than for the shallow materials. However, unlike in Experiment 1, post-hoc testing also suggested an absence of discernible differences in accuracy for deep versus shallow materials during the first response, $t(30)<1$.

Individual differences model predictions Turning to the outcome correlations, Table 4 again confirms the model's predictions. Although the correlations between raw gains and overall performance are now generally less negative (consistent with the speeding shift observed in Fig. 5), in every case the conditional gains have a markedly positive correlation with overall performance that is significantly more positive than that of the accompanying raw-gain correlation. In other words, as one goes from raw to conditional gain rates, the correlation with performance shifts from modestly negative (or slightly numerically positive, for deep materials) to robustly positive. This shift was significant for all three material types, as is indicated by the confidence intervals, and it was also significant when the data were collapsed across material types.

Because participants were sped more in Experiment 2 than in Experiment 1, Fig. 5 anticipates that the raw-gain correlations with overall performance would be less negative, as they are in every cell of Table 3 versus Table 4 . Nonetheless, these differences are not significant across experiments for any individual comparison, which itself is unsurprising, given the variability shown in Fig. 5. Thus, whereas the rightward shift in the gain correlation coefficients in Experiment 2 versus Experiment 1 is suggestive of another confirmed prediction of the noisy-accumulation model, larger sample sizes or within-subjects speeding manipulations would be necessary to convincingly confirm the prediction that increased speeding will shift the raw-gain correlations rightward.

Turning to losses, both the raw and conditional loss rates are strongly negatively correlated with overall performance. The conditional-loss correlation is reliably more negative than the raw-loss correlation for both shallow and deep materials, and trends in this direction for new materials and the collapsed data. This is consistent with the model predictions, in that the difference between the conditional- and raw-score correlations was anticipated to be smaller (Fig. 5), and hence somewhat less reliable, than the analogous gain findings. It is important to mention that the estimated confidence intervals take into account the dependence of the two correlations and that this is why even small absolute differences in the correlations can be reliable.

Finally, Table 4 confirms the prediction in Fig. 4 , in that the conditional losses are more tightly correlated with performance than the conditional gains. In every comparison, the absolute value of the correlation is larger for the former than for the latter.

Experiment 2 replicated Experiment 1 and confirmed the prediction that raw and conditional gain rates would diverge in their relationship to overall performance across observers. Moreover, the data were suggestive of another prediction of the model - namely, that with increased speeding, the correlation between raw gain rates and performance would become less negative (i.e., would shift rightward). However, larger samples or, preferably, within-subjects speeding manipulations will be required to definitively test this secondary

Table 4 Correlations of both gains and losses with overall performance in Experiment 2

\begin{tabular}{|c|c|c|c|c|c|c|c|c|c|c|c|}
\hline Items & Gain_r $r$ & $\mathrm{Cdl} \_$gain_r $r$ & $95 \% \mathrm{CI}$ & & Loss_r $r$ & Cdl_loss_r & $95 \% \mathrm{CI}$ & & Initialpc & Finalpc & $N$ \\
\hline New & -.18 & .65 & .6 & 1.25 & -.81 & -.81 & -.15 & .02 & .59 & .93 & 31 \\
\hline Shal & -.13 & .68 & .54 & 1.08 & -.64 & -.79 & -.29 & -.06 & .78 & .83 & 29 \\
\hline Deep & .07 & .83 & .47 & 1.21 & -.82 & -.88 & -.12 & -.01 & .78 & .94 & 31 \\
\hline All & -.23 & .69 & .62 & 1.26 & -.85 & -.91 & -.14 & 0 & .72 & .89 & 31 \\
\hline
\end{tabular}

95\% confidence intervals are for the differences between the conditional and raw correlation coefficients. Gain $r=$ correlation between raw gain rate and proportion correct, Cdl_gain_r $r=$ correlation between conditional gain rate and proportion correct, Loss_ $r=$ correlation between raw loss rate and proportion correct, Cdl_ loss $\bar{r}=$ correlation between conditional loss rate and proportion correct, Initialpc $=$ proportion of correct first responses, Finalpc $=$ overall proportion correct. For correlations involving conditional gains, one participant had a value of NA for shallow materials, and three had a value of NA for deep materials. This occurs whenever misses do not occur on the first response, and hence miss-miss + gains (miss-hit) in the denominator is undefined. 
prediction. Finally, the data confirmed the prediction that conditional loss rates are a more reliable indicator of differences in overall accuracy than are conditional gain rates, although both are strongly predictive of overall skill.

\section{General discussion}

The present data represent the first attempt to use a noisyaccumulation framework to make predictions at the level of individual differences in recognition reversal behavior. The data demonstrate that the raw gain rates of observers are not positively predictive, but instead are mildly negatively predictive of overall performance; observers who have frequent corrective responses are less accurate overall. In contrast, once one conditionalizes the gain rate on the possibility of making a correction (i.e., on the first-response miss rate), then gains become highly predictive of observer accuracy. This divergence occurs because increases in drift rates have opposing influences on the raw gain rate. Increasing drift rates reduce the tendency to miss on the first response (lowering the raw gain rate) but increase the tendency to correct misses that do occur on the first response (increasing both the raw and conditional gain rates). This results in a flat-tonegative relationship between raw gains and performance, but a clear positive relationship between conditional gains and performance. Relatedly, the model anticipates that although conditional gains will be clearly positively linked to performance, this relationship will be not as strong as the coupling of conditional losses to performance. Thus, the mechanics of this extremely basic noisy-accumulation model anticipate fundamentally different behavior for how gains and losses will link to individual differences in overall skill.

\section{Dual-process considerations}

The simulation predictions were based on a rudimentary noisy-accumulation model of judgment that used the signed mean evidence values in two serial epochs to render predictions. However, the model is essentially agnostic with respect to the number of retrieval processes occurring during recognition, because it only assumes that the net evidence increases across a trial. This evidence could represent a single source of mnemonic information, or it could reflect an amalgam of two or more sources (e.g., recollection and familiarity). Neither possibility conflicts with the idea that total evidence accrues during the course of an individual trial. Moreover, even if one assumes that recollection makes a later contribution to judgment than does familiarity during the course of a trial, as long as both contribute somehow during the two judgments, the present model remains tenable. Thus, the present predictions and findings are not suited to making single- versus dualprocess claims about recognition judgment (cf. Boldini et al., 2004)

\section{The need for dynamic models when considering rapid reversal behavior}

The present data demonstrate a characteristic pattern of reversal behavior consistent with noisy evidence accumulation. As we noted in the introduction, most decision models of recognition are "static," in that they do not explicitly characterize the evolution of evidence within a trial (Wixted \& Mickes, 2010; Yonelinas et al., 1996). If one is attempting to characterize asymptotic behavior for single judgments, this is not problematic. In contrast, in the present report we were interested in the manner in which participants rapidly reverse recognition conclusions, as appears to happen sometimes outside the laboratory, when passing an individual, for example. These experiences are symmetric, in that sometimes one greets a stranger warmly and only subsequently realizing the person is unknown (a gain for a new stimulus), or instead fails to acknowledge an acquaintance and only realizes too late that one should have greeted the individual (a gain for an old stimulus). Whether gains actually outnumber losses in such situations is an interesting question, but it is a natural applied prediction from this framework. Regardless, under static models these types of reversals are not easy to interpret. For example, although it is not directly incorporated in the relevant dual-process decision models, dual-process researchers often assume that recollection is available later in the trial than is familiarity. Thus, one explanation for why gains outnumber losses is that observers can sometimes correct an initial miss by using later-arriving recollection information. However, this does not explain why gains considerably outnumber losses for new materials, for which later-arriving recollection is not possible, nor does it readily explain why the raw gain rate is negatively related to overall performance for old materials. That is, if raw gains reflect the observer's use of recollection during the second response to correct familiarity-based misses, one might incorrectly predict that raw gain rates (being recollection-dependent) would anticipate high overall recognition performance, consistent with the idea of frequent recollection. Moreover, the notion of a latearriving recollection signal does not explain loss behavior.

Critically, the fact that a basic noisy-accumulation model anticipates patterns in rapid reversal behavior does not mean that it is well-suited to understanding asymptotic, self-paced recognition judgment and the various dissociations that are supportive of dual-process models of recognition memory (Diana, Reder, Arndt, \& Park, 2006; Yonelinas, 2002), because it is not designed to relate putative retrieval processes to differences in subjective experiences, neural structures, or task demands.

Although the rudimentary model used here and shown in Fig. 1 makes testable predictions about the relationships between reversal behaviors and overall recognition skill, it is a highly simplified approximation of the full drift-diffusion model. The important benefit of this simplification was that it enabled clear predictions about the relationships between reversal behavior and individual differences in skill, because those predictions were tied 
very tightly to the behavior of the evidence variable itself, by simply assuming that responses would track the mean of the evidence within a given epoch. However, this simplified model cannot accommodate phenomena such as speed-accuracy tradeoffs, because it does not generate reaction times for each trial. That is, the model we simulated assumes that observers can access the mean signal, or use a decision process sensitive to the mean signal, within particular epochs. However, it does not specifically model how they do this.

In the case of fully developed sequential-sampling models, a boundary decision rule is usually used. Thus, reaction times reflect when the cumulative evidence signal strikes an upper and/or a lower decision threshold value. This, combined with trial-to-trial variability in the evidence paths, guarantees that these models will always produce right-skewed reaction time distributions across trials, which are what is observed in empirical data (see, e.g., Ratcliff \& McKoon, 2008). However, this also means that these models assume that observers have limited control in regulating when to respond. Such models can speed responding generally by lowering the overall boundary for responding across trials (weak temporal control), but they cannot ensure that evidence is interrogated by a particular deadline on each trial (strong temporal control) when using a boundary rule. This is interesting because the response signal paradigm and other speeded-response procedures seem to assume, at least tacitly, that observers exercise strong temporal control over their reports in order to generally avoid late responses (for a brief discussion, see Ratcliff, 1978). Although it is perhaps not generally appreciated, this means that speeded response procedures may actually anticipate left- rather than right-skewed reaction time distributions. That is, if observers can regulate the time of responding while attempting to gather as much evidence as possible prior to the deadline, the reaction times might be bunched up against the response deadline, with a tail extending toward lower, quicker responses. In the present data, we evaluated the skew of each participant's correct initial responses in both Experiments 1 and 2. In Experiment 1, the skew was neither reliably positive nor negative across participants, $t(21)=$ $0.75, p=.460$. In Experiment 2, the data were reliably leftskewed, $t(30)=-4.42, p<.001$. However, in the present design we did not collect reaction times for late responses past the response deadline, so the absence of a right skew in Experiment 1 and the presence of a reliable left skew in Experiment 2 may simply be artifacts of using truncated reaction time distributions, and not evidence of the exercise of strong temporal control over responding.

Given the absence of right skew in the present data, future response signal research should use designs in which all reaction times (even those exceeding the deadline) are collected, perhaps in combination with time-out penalties and the temporal-signaling procedures used here. This would allow not only assessment of the full reaction time distribution for skew, but also post-hoc fitting of fully developed models. As we noted above, these models must produce right-skewed reaction time distributions, so one could test whether the empirical reaction time distributions demonstrated the appropriate degrees of rightward skew anticipated under the sequential-sampling models using boundary decision rules. If observers exercise strong temporal control of judgment timing, the empirical data might not.

Another potentially useful avenue for future research might be to compare different sequential-sampling decision models in their abilities to accommodate the relationships between reversal behavior and overall skill levels. The present study demonstrated that the most basic elements of the DDM anticipate several relationships between reversal behavior and individual differences in overall recognition skill. These present predictions are inherently tied to the within-trial, moment-to-moment noise process that is a central element of the DDM. However, an alternative choice model, the linear ballistic accumulator (LBA), does not assume any noise in the accumulation of evidence within each trial. Instead, it generates errors and reaction time predictions through trial-to-trial fluctuations in the starting point of evidence accumulation and the sampled drift rates of two competing accumulators, representing the correct and incorrect conclusions. ${ }^{3}$ Under this framework, correct reversals or gains presumably reflect trials on which the incorrect accumulator started with a higher signal (starting point variability), but the sampled drift rate was higher for the correct accumulator. Thus, gains require that the paths of the two evidence variables cross after the initial erroneous judgment, which would be the result of erroneously starting with more evidence for the incorrect accumulator. Thus, variations in the starting points across the two accumulators and variations in the drift rates across trials are fundamental for gains to occur in the two-response paradigm under the LBA. What remains to be considered is whether this framework can yield the negative relationship between raw gains and observer skill evidenced here, along with the other observations, such as the greater positive correlation between conditional loss rates and observer skill than between conditional gain rates and skill. However, directly comparing models in this fashion would require formal development of the decision process used to translate the evidence variables into two serial judgments during two-choice procedures. As we noted above, a critical first step should be to verify that during highly speeded response signal procedures, observer reaction times should nonetheless remain reliably right-skewed. This would suggest weak temporal control, consistent with a decision boundary being used for the first judgments in such paradigms.

\footnotetext{
${ }^{3}$ The DDM has been extended to incorporate variability in both starting point and drift rate across trials, so this calculation is not unique to the LBA. Nonetheless, the DDM does not require either form of variability to produce the reversal predictions, whereas both would be necessary for the LBA to address reversal behavior.
} 


\section{Other domains and predictions}

Although we focused on rapid reversals of recognition memory judgments, the present predictions also apply to nonmemory domains. For example, perceptual judgments, such as gender identification or movement discrimination, should display the same relationships. Thus, one avenue for future research will be to test this prediction in other judgment domains, to verify whether it is a general consequence of noisy evidence accumulation during speeded judgments. Moreover, some aspects of the basic model developed here remain to be tested. For example, the present framework assumes that there are no negative consequences from the initial judgment on the final judgment, because evidence continues to accumulate in the same fashion regardless of whether an initially speeded judgment is or is not rendered. That is, the level of evidence assessed on the second, unspeeded judgment is assumed not to be altered by performing the initial, speeded judgment. This constitutes a pretty strong assumption that could be tested by mixing the present two-response procedure with single, unspeeded trials or blocks for the same observers. The key question would be whether the final accuracy for the two-response trials matched that of the single, unspeeded response trials, as is assumed in the present framework.

\section{Conclusion}

The pattern of response reversals during recognition is consistent with a basic noisy evidence accumulation process at the level of outcomes (gains outnumber losses) and individual differences. With respect to the latter, raw gains are mildly negatively associated with recognition skill. That is, participants who demonstrate more corrective reversals are less accurate overall than those who demonstrate fewer corrective reversals, a pattern linked to the opposing effects of increasing drift rates on the raw frequencies of gains. In contrast, once the gains are conditionalized on the opportunity to observe a correction, there is a clear positive link between observer gain behavior and individual differences in accuracy. In other words, once the gain rate is translated into a probability of correction given that an error has occurred, it becomes highly predictive of individual differences in accuracy. Not only is this divergence of the raw and conditional gain rates anticipated by the noisy-accumulation framework, this same framework correctly predicts that the raw and conditional loss rates will not diverge similarly. Finally, the model correctly predicts that even when both gains and losses are conditionalized, the latter will be more predictive of individual differences in skill than will the former. Thus, the basic mechanics of noisy accumulation indicate that gains will outnumber raw losses, and that skilled recognizers will demonstrate infrequent corrections but with a high probability of correction should an initial error occur. Moreover, when ranking individuals, their conditional loss behavior should be a more sensitive indicator than their conditional gain behavior. The present empirical data are the first to demonstrate these anticipated individual difference patterns.

Author note This work was supported by National Institutes of Health Grant MH07398.

\section{Appendix: Correlation between drift rate and proportion correct}

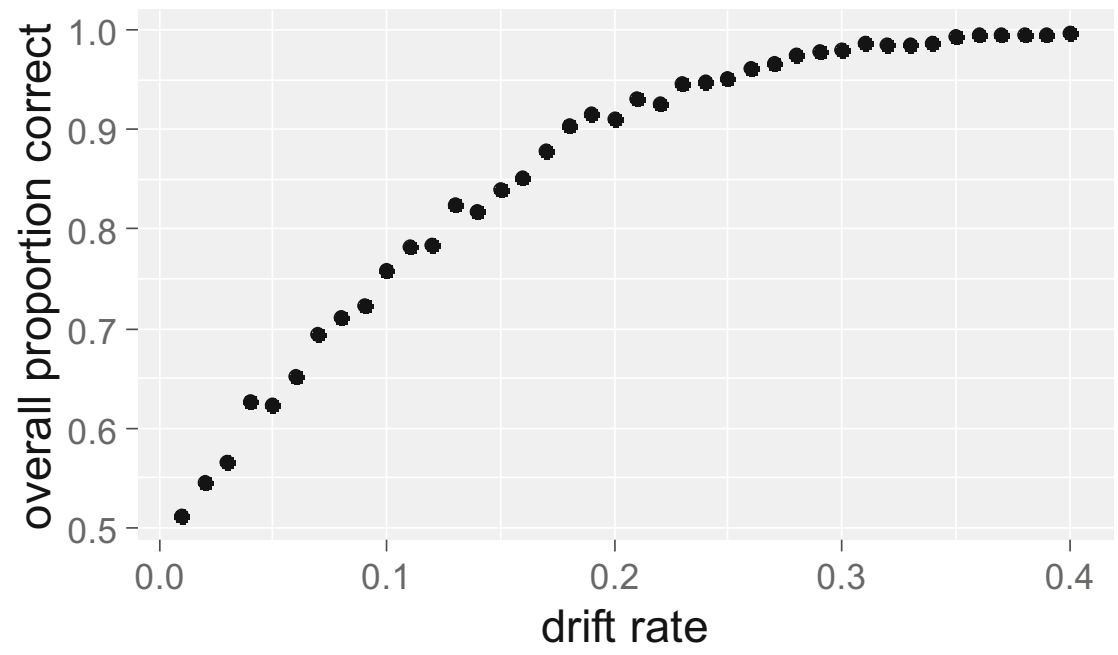




\section{References}

Benjamin, A. S., \& Craik, F. I. M. (2001). Parallel effects of aging and time pressure on memory for source: evidence from the spacing effect. Memory \& Cognition, 29, 691-697.

Boldini, A., Russo, R., \& Avons, S. E. (2004). One process is not enough! A speed-accuracy tradeoff study of recognition memory. Psychonomic Bulletin \& Review, 11, 353-361. doi:10.3758/BF03196582

Boldini, A., Russo, R., Punia, S., \& Avons, S. E. (2007). Reversing the picture superiority effect: A speed-accuracy trade-off study of recognition memory. Memory \& Cognition, 35, 113-123. doi:10.3758/BF03195948

Brown, S. D., \& Heathcote, A. (2008). The simplest complete model of choice reaction time: Linear ballistic accumulation. Cognitive Psychology, 57, 153-178. doi:10.1016/j.cogpsych.2007.12.002

Curran, T., DeBuse, C., \& Leynes, P. A. (2007). Conflict and criterion setting in recognition memory. Journal of Experimental Psychology: Learning, Memory, and Cognition, 33, 2-17.

Diana, R. A., Reder, L. M., Arndt, J., \& Park, H. (2006). Models of recognition: A review of arguments in favor of a dual-process account. Psychonomic Bulletin \& Review, 13, 1-21. doi:10.3758/BF03193807

Donkin, C., Averell, L., Brown, S., \& Heathcote, A. (2009). Getting more from accuracy and response time data: Methods for fitting the linear ballistic accumulator. Behavior Research Methods, 41, 1095-1110. doi:10.3758/BRM.41.4.1095

Dosher, B. A. (1984). Discriminating preexperimental (semantic) from learned (episodic) associations: A speed-accuracy study. Cognitive Psychology, 16, 519-555.

Espinosa-García, M., Vaquero, J. M. M., Miliken, B., \& Tudela, P. (2017). Recollection and familiarity for words and faces: A study comparing Remember-Know judgements and the Process Dissociation Procedure. Memory, 25, 19-34. doi:10.1080/ 09658211.2015.1120310

Gronlund, S. D., Edwards, M. B., \& Ohrt, D. D. (1997). Comparison of the retrieval of item versus spatial position information. Journal of Experimental Psychology: Learning, Memory, and Cognition, 23, 1261-1274. doi:10.1037/0278-7393.23.5.1261

Gronlund, S. D., \& Ratcliff, R. (1989). Time course of item and associative information: Implications for global memory models. Journal of Experimental Psychology: Learning, Memory, and Cognition, 15, 846-858.

Hautus, M. J. (1995). Corrections for extreme proportions and their biasing effects on estimated values of $d^{\prime}$. Behavior Research Methods, Instruments, \& Computers, 27, 46-51. doi:10.3758/BF03203619

Heit, E., Brockdorff, N., \& Lamberts, K. (2003). Adaptive changes of response criterion in recognition memory. Psychonomic Bulletin \& Review, 10, 718-723. doi:10.3758/BF03196537

Hintzman, D. L., \& Caulton, D. A. (1997). Recognition memory and modality judgments: a comparison of retrieval dynamics. Journal of Memory and Language, 37, 1-23.

Hintzman, D. L., Caulton, D. A., \& Levitin, D. J. (1998). Retrieval dynamics in recognition and list discrimination: Further evidence of separate processes of familiarity and recall. Memory \& Cognition, 29, 449-462.

Hintzman, D. L., \& Curran, T. (1997). Comparing retrieval dynamics in recognition memory and lexical decision. Journal of Experimental Psychology: General, 126, 228-247. doi:10.1037/0096-3445.126.3.228

Jacoby, L. L., \& Dallas, M. (1981). On the relationship between autobiographical memory and perceptual learning. Journal of Experimental Psychology: General, 110, 306-340. doi:10.1037/0096-3445.110.3.306

Johnson, M. K., Kounios, J., \& Reeder, F. A. (1994). Time-course studies of reality monitoring and recognition. Journal of Experimental Psychology: Learning, Memory, and Cognition, 20, 1409-1419.

Jones, T. C. (2006). Editing (out) generated study words in a recognition exclusion task: Effects of response signal delay and generation procedure. Memory, 14, 712-729.
Kim, K., \& Glanzer, M. (1993). Speed versus accuracy instructions, study time, and the mirror effect. Journal of Experimental Psychology: Learning, Memory, and Cognition, 19, 638-652. doi:10.1037/ 0278-7393.19.3.638

Koriat, A., \& Goldsmith, M. (1996). Monitoring and control processes in the strategic regulation of memory accuracy. Psychological Review, 103, 490-517. doi:10.1037/0033-295X.103.3.490

Kučera, H., \& Francis, W. N. (1967). Computational analysis of presentday American English. Providence, RI: Brown University Press.

Mandler, G. (1980). Recognizing: the judgment of previous occurrence. Psychological Review, 87, 252-271. doi:10.1037/0033-295X.87.3.252

McElree, B., Dolan, P. O., \& Jacoby, L. L. (1999). Isolating the contributions of familiarity and source information to item recognition: A time course analysis. Journal of Experimental Psychology: Learning, Memory, and Cognition, 25, 563-582.

Pleskac, T. J., \& Busemeyer, J. R. (2010). Two-stage dynamic signal detection: A theory of choice, decision time, and confidence. Psychological Review, 117, 864-901.

Ratcliff, R. (1978). A theory of memory retrieval. Psychological Review, 85, 59-108. doi:10.1037/0033-295X.85.2.59

Ratcliff, R., \& McKoon, G. (2008). The diffusion decision model: Theory and data for two-choice decision tasks. Neural Computation, 20, 873-922. doi:10.1162/neco.2008.12-06-420

Ratcliff, R., Thapar, A., \& McKoon, G. (2004). A diffusion model analysis of the effects of aging on recognition memory. Journal of Memory and Language, 50, 408-424. doi:10.1016/j.jml.2003.11.002

Ratcliff, R., Thapar, A., \& McKoon, G. (2010). Individual differences, aging, and IQ in two-choice tasks. Cognitive Psychology, 60, 127 157. doi:10.1016/j.cogpsych.2009.09.001

Reddi, B. A., \& Carpenter, R. H. (2000). The influence of urgency on decision time. Nature Neuroscience, 3, 827-30.

Reed, A. V. (1973). Speed-accuracy trade-off in recognition memory. Science, 181, 574-579. doi:10.1126/science.181.4099.574

Rotello, C. M., \& Heit, E. (2000). Associative recognition: A case of recall-to-reject processing. Memory \& Cognition, 28, 907-922.

Snodgrass, J. G., \& Corwin, J. (1988). Pragmatics of measuring recognition memory: Applications to dementia and amnesia. Journal of Experimental Psychology: General, 117, 34-50. doi:10.1037/ 0096-3445.117.1.34

Starns, J. J., \& Ratcliff, R. (2010). The effects of aging on the speedaccuracy compromise: Boundary optimality in the diffusion model. Psychology and Aging, 25, 377-390.

Toth, J. P. (1996). Conceptual automaticity in recognition memory: Levels-of-processing effects on familiarity. Canadian Journal of Experimental Psychology, 50, 123-138.

Van Zandt, T., \& Maldonado-Molina, M. M. (2004). Response reversals in recognition memory. Journal of Experimental Psychology: Learning, Memory, and Cognition, 30, 1147-1166. doi:10.1037/ 0278-7393.30.6.1147

Wixted, J. T., \& Mickes, L. (2010). A continuous dual-process model of remember/know judgments. Psychological Review, 117, 10251054. doi:10.1037/a0020874

Yonelinas, A. P. (2002). The nature of recollection and familiarity: A review of 30 years of research. Journal of Memory and Language, 46, 441-517. doi:10.1006/jmla.2002.2864

Yonelinas, A. P., Dobbins, I., Szymanski, M. D., Dhaliwal, H. S., \& King, L. (1996). Signal-detection, threshold, and dual-process models of recognition memory: ROCs and conscious recollection. Consciousness and Cognition, 5, 418-441. doi:10.1006/ccog.1996.0026

Yonelinas, A. P., \& Jacoby, L. L. (1994). Dissociations of processes in recognition memory: Effects of interference and response speed. Canadian Journal of Experimental Psychology, 48, 516-535. doi: 10.1037/1196-1961.48.4.516

Yonelinas, A. P., \& Jacoby, L. L. (1996). Noncriterial recollection: Familiarity as automatic, irrelevant recollection. Consciousness and Cognition, 5, 131-141. 